\title{
Effect of Van Hove singularities in the onset of pseudogap states in Mott insulators
}

\author{
Wei Wu, ${ }^{1,2,3}$ Mathias S. Scheurer, ${ }^{4}$ Michel Ferrero $\odot,{ }^{1,3}$ and Antoine Georges ${ }^{1,3,5,6}$ \\ ${ }^{1}$ CPHT, CNRS, École Polytechnique, IP Paris, F-91128 Palaiseau, France \\ ${ }^{2}$ School of Physics, Sun Yat-sen University, Guangzhou, Guangdong Province 510275, China \\ ${ }^{3}$ Collège de France, 11 place Marcelin Berthelot, 75005 Paris, France \\ ${ }^{4}$ Department of Physics, Harvard University, Cambridge, Massachusetts 02138, USA \\ ${ }^{5}$ Center for Computational Quantum Physics, Flatiron Institute, 162 Fifth Avenue, New York, New York 10010, USA \\ ${ }^{6}$ DQMP, Université de Genève, 24 quai Ernest Ansermet, CH-1211 Genève, Switzerland
}

(Received 26 January 2020; revised manuscript received 7 June 2020; accepted 11 June 2020; published 14 July 2020)

\begin{abstract}
The Mott insulating phase of parent compounds is frequently taken as a starting point for underdoped high- $T_{c}$ cuprate superconductors. In particular, the pseudogap state is often considered as deriving from the Mott insulator. In this work, we systematically investigate different weakly doped Mott insulators on a square and triangular lattice to clarify the relationship between the pseudogap and Mottness. We show that doping a two-dimensional Mott insulator does not necessarily lead to a pseudogap phase. Despite its inherent strongcoupling nature, we find that the existence or absence of a pseudogap depends sensitively on noninteracting band parameters, and we identify the crucial role played by the van Hove singularities of the system. Motivated by a $\mathrm{SU}(2)$ gauge theory for the pseudogap state, we propose and verify numerically a simple equation that governs the evolution of characteristic features in the electronic scattering rate.
\end{abstract}

DOI: 10.1103/PhysRevResearch.2.033067

\section{INTRODUCTION}

The complex phenomenology of cuprate high- $T_{c}$ superconductors is widely thought of as a consequence of introducing mobile charge carriers in a Mott insulator through doping [1]. In the regime of low doping, a pseudogap (PG) state [2-4] prevails below an onset temperature $T^{*}$, which lies above the superconducting dome. There are a number of theories proposed to describe the dominant physics in this regime, such as preformed Cooper pairs [5], various emergent ordered states [6-9], topological order [10-12], and Mottness collapse [13-17]; however, to date, a full consensus has not been reached. On the numerical side, prototypical models such as the two-dimensional Hubbard model $[18,19]$ or the $t-J$ model [20,21] have been investigated intensively. Regarding the origin of the PG state, different approaches point to shortrange antiferromagnetic (AF) correlations and proximity to Mottness, including quantum Monte Carlo [22-24], exact diagonalization [20], cluster perturbation theory [25], and cluster extensions of dynamical mean-field theory (DMFT) [26-31]. This is in line with a recent systematic experimental study [32] indicating that all other instabilities are secondary to AF correlations in the opening of the PG.

Although the importance of AF correlations and Mottness is stressed by various studies, the exact underlying mechanism

Published by the American Physical Society under the terms of the Creative Commons Attribution 4.0 International license. Further distribution of this work must maintain attribution to the author(s) and the published article's title, journal citation, and DOI. of the PG is still an open question. It is natural to expect that long-range AF fluctuations can cause a PG due to the coupling of the electrons to collective magnetic modes [33-36]. In hole-doped cuprates, however, this picture does not apply, as the correlation length is significantly reduced. To shed light on the role played by AF correlations and Mottness, we address in this work the following fundamental question: do all doped Mott insulators with short-range AF correlations have a $P G$ in two dimensions? To this end, we systematically study Hubbard models on square and triangular lattices (see Fig. 1), which have very different magnetic frustration properties. We discover that, surprisingly, on both lattices the existence of a PG at finite doping in the strong-coupling regime depends sensitively on the electronic dispersion of the noninteracting system. Numerical evidence suggests that this dependence can be well described by a simple relation that involves the location of the van Hove singularity (VHS). In addition, we find a simple equation that captures the evolution of the "quasipole" [31] in the electronic scattering rate, which is related to the depletion of the spectral weight in the PG. This equation agrees with an SU(2) gauge theory [10-12], where the PG is the result of a Higgs mechanism, physically corresponding to local AF order with large orientational fluctuations.

\section{MODEL AND METHOD}

We use the dynamical cluster approximation (DCA) [27], a cluster extension of single-site DMFT [26], to study the triangular- and square-lattice Hubbard models. The DCA method can effectively capture temporal and short-ranged spatial correlations by mapping the original lattice problem onto a quantum cluster model embedded in an effective medium 

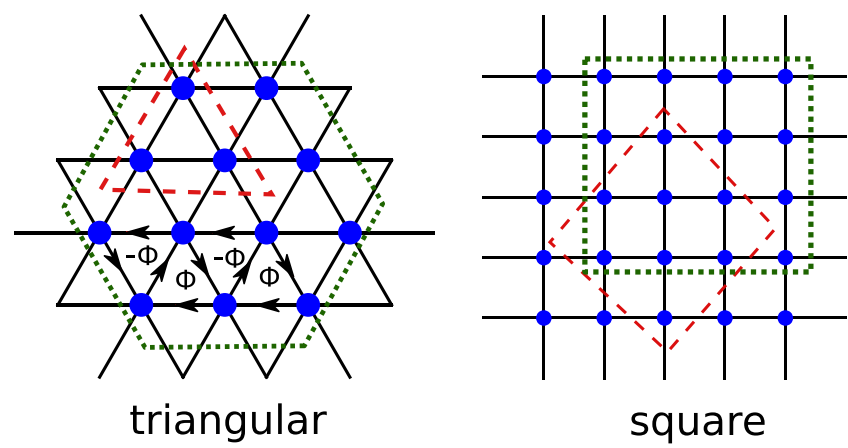

FIG. 1. Illustration of triangular- (left) and square- (right) lattice tight-binding models studied in this work. Here we study complex nearest-neighbor hopping on the triangular lattice with $-t e^{+i \Phi / 3}$ (along arrow) or $-t e^{-i \Phi / 3}$ (against arrow); electrons encircling a triangular plaquette once acquire a phase of $\pm \Phi$ as indicated. The geometries of the DCA clusters we use are indicated by red and green dashed lines.

[26]. The DCA clusters we use are illustrated in Fig. 1. For concreteness, we focus on an on-site Hubbard interaction, $U$, and the corresponding Hamiltonians have the form

$$
H=-\sum_{i, j, \sigma} t_{i j} c_{i \sigma}^{\dagger} c_{j \sigma}+U \sum_{i} n_{i \uparrow} n_{i \downarrow}-\mu \sum_{i, \sigma} n_{i \sigma},
$$

where $c_{i \sigma}^{\dagger}$ are electronic creation operators on site $i$ with spin $\sigma=\uparrow, \downarrow, n_{i \sigma}=c_{i \sigma}^{\dagger} c_{i \sigma}$, and $\mu$ denotes the chemical potential. The magnitude of hopping between nearest-neighbor sites, $t$, defines the energy unit throughout this paper, $t \equiv 1$. Note that for a triangular lattice we use the direction-dependent hopping $t_{i j}=t e^{ \pm i \Phi / 3}$, which corresponds to a staggered magnetic flux threading the triangular plaquettes [37]; see Fig. 1, left panel. By changing the phase factor $\Phi$, one can systematically vary the dispersion relation while the antiferromagnetic exchange to dominant order in $t / U$ is unaffected. Hence, the Heisenberg model and magnetic correlations associated with model (1) at strong coupling are independent of $\Phi$, which enters only at subleading orders in $t / U$ by inducing chiral terms [38]. We note in passing that studying doped triangular lattice models with flux is further motivated by their relevance as minimal models of moiré superlattice systems $[39,40]$, where opposite valleys have opposite flux due to time-reversal symmetry.

\section{TRIANGULAR LATTICE}

We first show three-site DCA results on the triangular lattice in Fig. 2. At half-filling, we find that the triangular lattice model without flux, $\Phi=0$, becomes insulating for sufficiently strong interactions $U>U_{c}^{\text {Mott }} \simeq 8.2$ at $T=0.06-$ in good agreement with previous numerical results [41-43]. In the following, we take $U=9.2$, which corresponds to $U \simeq$ $1.15 U_{c}^{\text {Mott }}$ and to the regime of moderately strong correlations at which a chiral spin liquid phase was found at half-filling in [43] (before the spins order for $U>10.6$ ). A characteristic feature of the PG state is the opening of a partial energy gap in the spectral function $A(\boldsymbol{k}, \omega)$ near the Fermi level, at certain regions in the Brillouin zone (BZ). As can be seen in Fig. 2(a), $A\left(\boldsymbol{k}_{F}, \omega\right)$ at a typical momentum $\boldsymbol{k}_{F}$ on
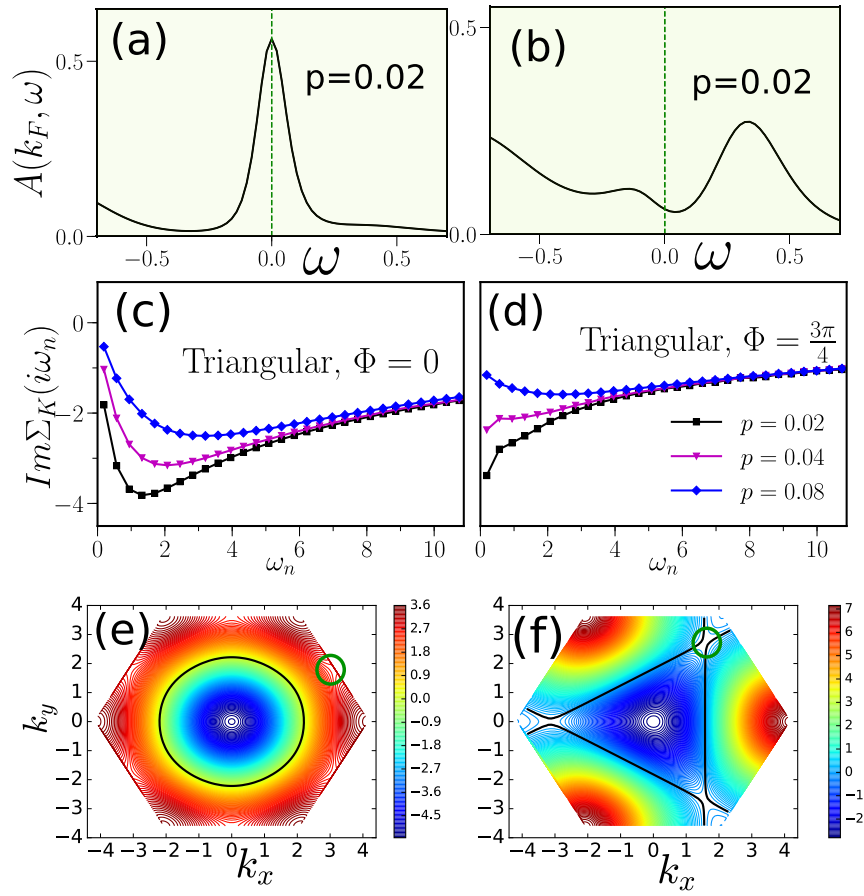

FIG. 2. Dependence of the PG on the bare dispersion for the triangular lattice. In (a) and (b), we show the spectral function of the triangular lattice model at low hole doping $p=0.02$ for $\Phi=0$ [no PG, $\left.\boldsymbol{k}_{F}=(1.3,2.2)\right]$ and $\Phi=3 \pi / 4$ [with PG, $\boldsymbol{k}_{F}=\left(0.5, \frac{2 \sqrt{3}}{3} \pi\right)$ ], respectively. The imaginary part of the corresponding cluster selfenergies for these values of $\boldsymbol{k}_{F}$ [both belong to a DCA patch centered at $\left.\boldsymbol{k}=\left(\frac{2 \pi}{3}, \frac{2 \sqrt{3}}{3} \pi\right)\right]$ is plotted as a function of Matsubara frequency for a few different dopings in (c) and (d). In (e) and (f), we show the Hartree-shifted dispersions $\epsilon_{k}^{*}=\epsilon_{k}-\mu+\langle n\rangle U / 2$ (color plot) at $p=0.04$ together with the resulting $\epsilon_{k}^{*}=0$ surface (black solid line) for $\Phi=0$ and $\Phi=3 \pi / 4$. Furthermore, the VHSs are indicated by green circles. In all plots, we take $T=0.06, U=1.15 U_{c}^{\text {Mott }}$, i.e., $U \simeq 9.5(U \simeq 7.7)$ for $\Phi=0(\Phi=3 \pi / 4)$.

the Fermi surface [defined by $\operatorname{Re} G\left(\boldsymbol{k}_{F}, \omega=0\right)=0$ ] of the slightly doped triangular lattice (hole doping $p=0.02$ ) with $\Phi=0$ shows a well-defined quasiparticle peak; no evidence of a PG is seen over the whole BZ. Nevertheless, when one turns on the staggered magnetic flux, the spectral function becomes qualitatively different from its $\Phi=0$ counterpart: as can be seen in Fig. 2(b), a PG reappears for $\Phi=3 \pi / 4$ (same doping $p=0.02$ and even smaller $U$ to keep $U / U_{c}^{\text {Mott }}$ the same).

To gain further insights into this surprising difference, Figs. 2(c) and 2(d) show a direct comparison of the imaginary part of the self-energy $\Sigma\left(\boldsymbol{k}, i \omega_{n}\right)$ with $\Phi=0$ and $\Phi=3 \pi / 4$ for different $p$. We see that the self-energy at $\Phi=3 \pi / 4$ inherits an insulating-like behavior from the half-filled Mott insulator for small, finite $p$, characterized by an enhanced amplitude of scattering at small frequencies. A metallic $\Sigma\left(\boldsymbol{k}, i \omega_{n}\right)$ is only found for significant doping $(p \geqslant 0.08)$. In contrast, for $\Phi=0$, there is a sudden loss of low-energy scattering when the Mott insulator is doped [44], as the self-energy immediately turns metallic upon doping. This is in accordance with $A\left(\boldsymbol{k}_{F}, \omega\right)$ in Fig. 2(a), where no PG was observed either. Note that the absence of a PG on the triangular lattice $(\Phi=0)$ 


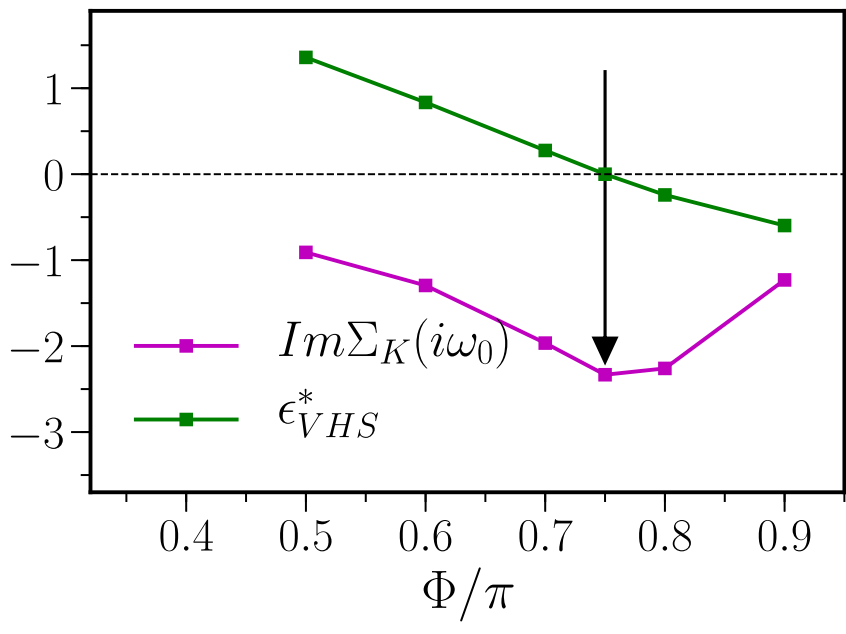

FIG. 3. By continuously changing the bare dispersion of the triangular lattice via $\Phi$, we find that the strongest low-energy scattering, indicated by the most negative imaginary part of the self-energy at the first Matsubara frequency, is found [at $K=\left(\frac{2 \pi}{3}, \frac{2 \sqrt{3}}{3} \pi\right)$ ] when the value $\epsilon_{\boldsymbol{k}}^{*}$ at the momentum $\boldsymbol{k}=\boldsymbol{k}_{\mathrm{VHS}}$ of the VHS, $\epsilon_{\mathrm{VHS}}^{*}$, vanishes. Here we use a three-site DCA, $U=8, T=0.08$, and $p=0.04$.

was also noticed previously [45], where the author attributes it to the triangular lattice being too frustrated. Here, the shown strong sensitivity of PG to staggered flux $\Phi$ suggests it is not the degree of geometric frustration that controls whether a PG is present or not.

One might wonder whether there is a simple way to understand how $\Phi$ affects the PG. To address this question, we plot in Figs. 2(e) and 2(f) the bare dispersions $\epsilon_{k}$ with chemical potential and Hartree shift subtracted,

$$
\epsilon_{\boldsymbol{k}}^{*}=\epsilon_{\boldsymbol{k}}-\mu+U\langle n\rangle / 2,
$$

for $\Phi=0$ and $\Phi=3 \pi / 4$, respectively. For $\Phi=0$, the VHS, indicated by the green circle, is far away from the line where $\epsilon_{k}^{*}=0$ (black line); note that this is not the bare, noninteracting Fermi surface and not the interacting one either, which would require the self-energy in Eq. (2). In the following, we will refer to this surface as the " $\epsilon_{\boldsymbol{k}}^{*}=0$ surface." We observe that the VHS is located almost exactly at the $\epsilon_{k}^{*}=0$ surface when $\Phi=3 \pi / 4$. We also found that when $\Phi$ is continuously changed, the enhancement of low-energy scattering is essentially guided by the VHS approaching the $\epsilon_{k}^{*}=0$ surface, as suggested by the self-energy plot in Fig. 3. This indicates that the position of the VHS relative to the $\epsilon_{k}^{*}=0$ surface plays a crucial role in the formation of the PG. This observation does not only apply on the triangular lattice: on the square lattice, the enhancement of the PG is also guided by the VHS reaching the $\epsilon_{k}^{*}=0$ surface, as we will see next.

\section{SQUARE LATTICE}

We now study the square lattice with nearest-, $t \equiv 1$, and next-nearest-neighbor hopping, $t^{\prime}$, which is a prototype model for the high- $T_{c}$ cuprates. Following the discussion of the triangular lattice, we use the spectral function and the imaginary part of the self-energy, shown in Figs. 4(a) and 4(b), to probe the PG for two different band parameters, $t^{\prime}= \pm 0.2$.
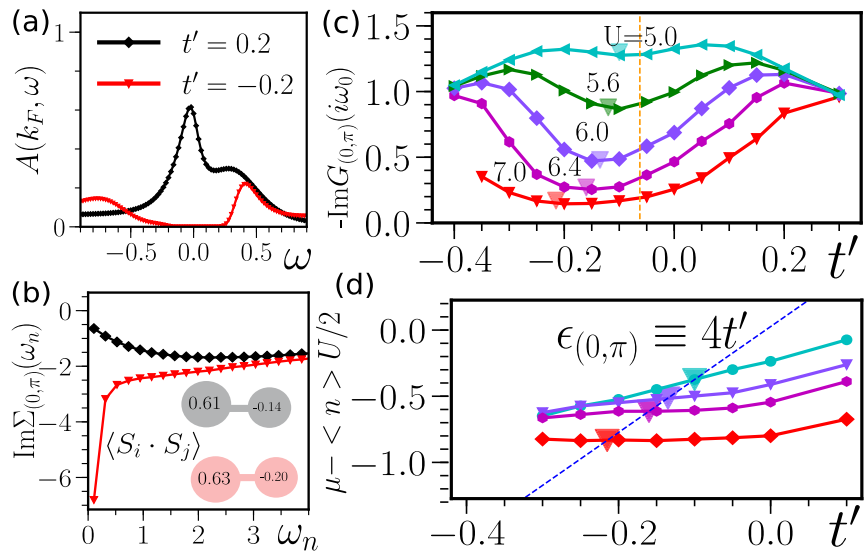

FIG. 4. PG and its parameter dependence on the square lattice at hole doping $p=0.05$ and $T=1 / 30$. (a) Spectral function at the Fermi surface for $t^{\prime}=0.2$ [black line, $\boldsymbol{k}_{F}=(3.0,0)$ ] and $t^{\prime}=-0.2$ [red line, $\boldsymbol{k}_{F}=(\pi, 0)$ ] for $U=7$. The corresponding imaginary part of the antinodal self-energy for the same set of parameters is shown as a function of Matsubara frequency in (b). The inset illustrates the on-site (left) and nearest-neighbor (right) spin-spin correlations using the same color code as in the main panels of (a) and (b). In (c), the imaginary part of the antinodal Green's function at the lowest Matsubara frequency is shown as a function of next-nearest-neighbor hopping $t^{\prime}$ for various $U$. The minimum of the spectral intensity in (c) corresponds to the maximum of the PG. The triangles indicate the value of $t^{\prime}$ at which the shifted chemical potential, solid lines in (d), is equal to $\epsilon_{(0, \pi)}=4 t^{\prime}$, blue dashed line in (d), i.e., when $\epsilon_{k}^{*}=\epsilon_{k}-\mu+\langle n\rangle U / 2=0$ at the VHS $k=(0, \pi)$.

It is worth noting that the two cases $t^{\prime}= \pm 0.2$ are actually identical Mott insulators at half-filling, related by a particlehole transformation. However, upon doping, the low-energy scattering properties quickly become different: as one can see in Figs. 4(a) and 4(b), the negative $t^{\prime}$ case has a well-developed PG and an insulatorlike self-energy, whereas positive $t^{\prime}$ leads to metallic behavior. This has been noticed before $[46,47]$, but the mechanism behind this striking asymmetry has remained unclear.

To provide a systematic approach, we show $-\operatorname{Im} G_{k}\left(i \omega_{n=0}\right)$ at the antinode $\boldsymbol{k}=(0, \pi)$ as a function of $t^{\prime}$ for different $U$ in Fig. 4(c). We recover that the antinodal quasiparticles are most suppressed for negative $t^{\prime}$. As $U$ increases, the value of $t^{\prime}$ with strongest suppression (marked by the triangles) becomes more and more negative. Most importantly, Fig. 4(d) reveals that the same triangles also correspond to the value of $t^{\prime}$ for which the VHSs [located at $\boldsymbol{k}_{\mathrm{VHS}}=(\pi, 0),(0, \pi)$ ] hit the $\epsilon_{k}^{*}=0$ surface, i.e., when $\epsilon_{k_{\mathrm{VHS}}}^{*}=0$. In other words, just like in the triangular-lattice model, the existence of a PG on the square lattice is determined by whether a VHS is close to the line of zeros of the renormalized dispersion $\epsilon_{k}^{*}$. In particular, this also explains the aforementioned particle-hole asymmetry regarding the PG: for $t^{\prime}=-0.2$, the VHSs are closer to the $\epsilon_{k}^{*}=0$ surface than for $t^{\prime}=0.2$.

A few comments are in order. We first note that this empirical rule for the presence of a PG also applies to different values of $U$ [different colors in Figs. 4(c) and 4(d)], different dopings, and holds for larger DCA cluster-sizes (16-site) or different types of hopping terms (third-nearest-neighbor 

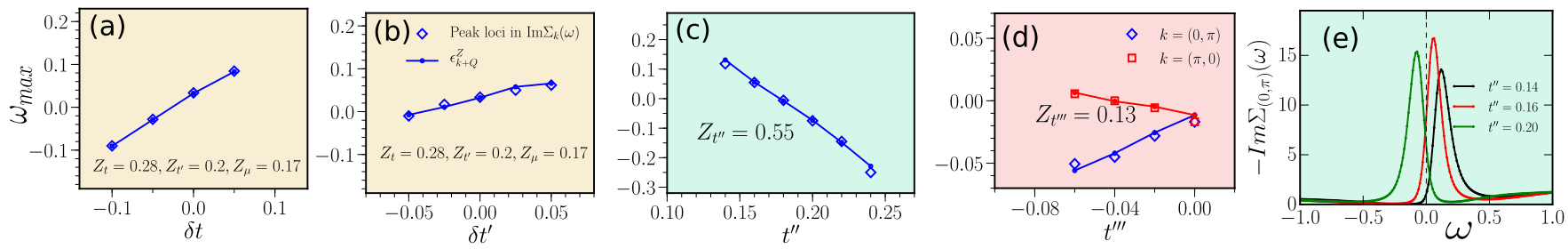

FIG. 5. Quasipole of antinodal self-energy can be described by Eq. (3). In (a)-(d), symbols show the frequencies $\omega_{\text {peak }}$ of the peaks in $\operatorname{Im} \Sigma_{k}(\omega)$ [at $\boldsymbol{k}=(0, \pi)$ unless otherwise specified] found by the maximum entropy method analytical continuation on $\Sigma_{k}\left(i \omega_{n}\right)$, while lines display $\epsilon_{k+Q}^{Z}-\mu^{\prime}$ with values of $Z$ marked in the corresponding plots. (a) and (b) Hubbard model with $\epsilon_{k}=-2\left[(t+\delta t) \cos \left(k_{x}\right)+\right.$ $\left.(t-\delta t) \cos \left(k_{y}\right)\right]-4\left(t^{\prime}+\delta t^{\prime}\right)\left[\cos \left(k_{x}\right) \cos \left(k_{y}\right)\right]$ with $t^{\prime}=-0.2$, tuning $\delta t$ and $\delta t^{\prime}$. For both plots, $U=7, p=0.08, T=1 / 30$. (c) $\epsilon_{k}=$ $-2 t\left[\cos \left(k_{x}\right)+\cos \left(k_{y}\right)\right]-4 t^{\prime}\left[\cos \left(k_{x}\right) \cos \left(k_{y}\right)\right]-2 t^{\prime \prime}\left[\cos \left(2 k_{x}\right)+\cos \left(2 k_{y}\right)\right]$ with fixed $t^{\prime}=0.1$ and changing $t^{\prime \prime}$. Here $U=6, p=0.05, T=$ 0.06. (d) $\epsilon_{k}=-2 t\left[\cos \left(k_{x}\right)+\cos \left(k_{y}\right)\right]-4 t^{\prime}\left[\cos \left(k_{x}\right) \cos \left(k_{y}\right)\right]-2 t^{\prime \prime \prime}\left[\cos \left(2 k_{x}\right) \cos \left(k_{y}\right)\right]$ with fixed $t^{\prime}=-0.2$ and changing $t^{\prime \prime \prime}$. Here $U=7, p=$ $0.05, T=1 / 30$. (e) Imaginary part of the antinodal self-energy as a function of frequency, where parameters are the same as for plot (c). Note that while (a), (b), and (d) show 8-site DCA results, (c) and (e) are from 16-site calculations.

hopping, for example; not shown here). Let us also stress that, as on the triangular lattice, the difference between $t^{\prime}=$ +0.2 and -0.2 cannot be attributed to magnetic properties: as can be seen in the inset of Fig. 4(b), both the on-site and nearest-neighbor magnetic correlations are approximately the same for the two values of $t^{\prime}$, although one has a metallic and the other one an insulating antinodal self-energy. We finally emphasize that our criterion involves the renormalized dispersion $\epsilon_{\boldsymbol{k}}^{*}$ in Eq. (2) rather than the bare $\epsilon_{\boldsymbol{k}}$. This seems plausible as diagrammatic studies [24] at half-filling show that the strong-coupling Feynman series converges when particle lines are expressed as $g_{0}^{-1}\left(\boldsymbol{k}, i \omega_{n}\right)=i \omega_{n}-\epsilon_{\boldsymbol{k}}^{*}$ and it diverges when using $g_{0}^{-1}\left(\boldsymbol{k}, i \omega_{n}\right)=i \omega_{n}-\epsilon_{\boldsymbol{k}}+\mu$ instead. This indicates that any perturbative treatment of PG physics should be based on $\epsilon_{k}^{*}$ rather than on the bare $\epsilon_{k}$. We emphasize, however, that the above analysis certainly cannot be perceived as a weak-coupling picture of VHS-enhanced quasiparticle scattering either: in the weak-coupling framework, the AF correlation length $\xi$ must be larger than the thermal de Broglie wavelength $[35,36]$ to obtain a PG at the "hotspot" of the Fermi surface. VHSs can enhance low-energy scattering, but the PG continues to exist even if all VHSs are removed from the system in the weak-coupling picture. In contrast, in our strong-coupling study, $\xi$ is only a few lattice spacings and the PG disappears in the absence of VHSs.

\section{QUASIPOLE OF SELF-ENERGY IN A PG REGIME}

So far we have been studying the prerequisites for the existence of a PG in underdoped Mott insulators. We will next discuss the property of strong antinodal scattering when the PG is present on the square lattice. There have been various numerical studies $[14,31,48]$ revealing that the antinodal self-energy has a low-energy quasipole in the PG regime [see Fig. 5(e)], connected to the opening of a PG in the spectral function. By changing the hopping parameters, here we monitor the evolution of the quasipole of the antinodal self-energy in the parameter regime where a strong PG is found. We display results for four different variations of the hopping parameters in Fig. 5 on a square lattice. Remarkably, we find that in all cases the variation of the peak locus of the self-energy in frequency space can be described by the simple relation

$$
\omega_{\text {peak }}(\boldsymbol{k})=\epsilon_{\boldsymbol{k}+Q}^{Z}-\mu^{\prime} .
$$

Here $\epsilon_{k}^{Z}$ is the bare dispersion with hopping terms renormalized by constants $Z$, and $\mu^{\prime}$ is the chemical potential with Hartree shift subtracted and rescaled by $Z_{\mu}$, i.e., $\mu^{\prime}=Z_{\mu}(\mu-\langle n\rangle U / 2)$. Furthermore, $\boldsymbol{Q}=(\pi, \pi)$ is the $\mathrm{AF}$ wave vector. The specific values of the renormalization constants depend on the physical parameters such as doping $p$ and interaction strength $U$. In Figs. 5(a) and 5(b) we show that, for the square lattice with nearest- and nextnearest neighbor hopping, $\omega_{\text {peak }}(\boldsymbol{k})$ for $\boldsymbol{k}=(0, \pi)$ is described by Eq. (3) with $\epsilon_{k}^{Z}=-2 Z_{t} t\left[\cos \left(k_{x}\right)+\cos \left(k_{y}\right)\right]-$ $4 Z_{t^{\prime}} t^{\prime}\left[\cos \left(k_{x}\right) \cos \left(k_{y}\right)\right], Z_{t}=0.28, Z_{t^{\prime}}=0.2$, and $Z_{\mu}=0.17$. Note that Eq. (3) is fulfilled in all our DCA computations with different clusters and different hopping terms. This is illustrated in Figs. 5(c) and 5(d).

While this behavior is essentially an empirical observation, the form of Eq. (3) is motivated by a (2+1)-dimensional SU(2) gauge theory proposed for the pseudogap phase [10-12]: in that theory, the electronic self-energy is peaked at the frequencies $\omega_{\text {peak }}^{\mathrm{SU}(2)}(\boldsymbol{k})=\epsilon_{\boldsymbol{k}+\boldsymbol{Q}}^{Z}-\mu$, where the self-energy of the "chargons," the charge carriers in the bulk of the system, has poles. For instance, $Z_{t} \simeq 0.3$ and $Z_{t^{\prime}} \simeq 0.2$ were found [10] in this description for the case of nearest- and next-nearestneighbor hopping, in good agreement with the coefficients obtained by DCA; see Figs. 5(a) and 5(b).

\section{CONCLUSION AND DISCUSSION}

In this work, we have systematically analyzed which conditions are favorable for the emergence of a PG when doping insulating half-filled Hubbard models. We identified empirically the proximity of VHSs to the $\epsilon_{k}^{*}=0$ surface, defined by $\epsilon_{\boldsymbol{k}}^{*}=0$ with renormalized dispersion $\epsilon_{\boldsymbol{k}}^{*}$ in Eq. (2), as favoring the formation of a PG. Theoretical clarification for why the VHSs are key in the strong-coupling regime is clearly needed. We believe that a systematic analysis of magnetic order and potential spin-liquid phases at the half-filled triangular lattice model with staggered flux could provide important missing information to understand the observed behavior. Inspired 
by an emergent gauge theory proposed for the pseudogap phase [10-12], we also verified the relation in Eq. (3) for the frequency $\omega_{\text {peak }}$ at which the self-energy exhibits a quasipole inside the pseudogap phase for several different bare dispersions on the square lattice. In experiments, the organic Mott insulator $\kappa-(\mathrm{BEDT}-\mathrm{TTF})_{2} \mathrm{Cu}\left[\mathrm{N}(\mathrm{CN})_{2}\right] \mathrm{Cl}$ can be modeled as a single-band Hubbard model, where strong doping asymmetry has been reported [49]. In agreement with our result shown in Fig. 4(c), it is indeed found that the interaction effect can be greatly enhanced as moving from the electrondoping [corresponding to $t^{\prime}>0$ cases in Fig. 4(c)] side to the hole-doping $\left(t^{\prime}<0\right)$ side [49]. Finally, we note that a highly tunable monolayer $\mathrm{Bi}_{2} \mathrm{Sr}_{2} \mathrm{CaCu}_{2} \mathrm{O}_{8+\delta}$ was recently fabricated in experiments [50]. The importance of $\epsilon_{\boldsymbol{k}}^{*}$ defined by Eq. (2) in pseudogap physics can then directly verified in this system in future experiments.

\section{ACKNOWLEDGMENTS}

We thank S. Sachdev and T. Senthil for valuable discussions. W.W. acknowledges the funding from Sun Yat-sen University (Grant No. 18841204) and the National Natural Science Foundation of China (Grant No. 41030053). M.S.S acknowledges support from the National Science Foundation under Grant No. DMR-1664842. This work was supported by a grant from the Swiss National Supercomputing Centre (CSCS) under project ID S820. The Flatiron Institute is a division of the Simons Foundation.
[1] P. A. Lee, N. Nagaosa, and X.-G. Wen, Doping a Mott insulator: Physics of high-temperature superconductivity, Rev. Mod. Phys. 78, 17 (2006).

[2] H. Ding, T. Yokoya, J. Campuzano, T. Takahashi, M. Randeria, M. Norman, T. Mochiku, K. Kadowaki, and J. Giapintzakis, Spectroscopic evidence for a pseudogap in the normal state of underdoped high-tc superconductors, Nature (London) 382, 51 (1996).

[3] T. Timusk and B. Statt, The pseudogap in high-temperature superconductors: An experimental survey, Rep. Prog. Phys. 62, 61 (1999).

[4] M. R. Norman, D. Pines, and C. Kallin, The pseudogap: Friend or foe of high $T_{c}$, Adv. Phys. 54, 715 (2005).

[5] T. Senthil and P. A. Lee, Synthesis of the phenomenology of the underdoped cuprates, Phys. Rev. B 79, 245116 (2009).

[6] S. A. Kivelson, E. Fradkin, and V. J. Emery, Electronic liquidcrystal phases of a doped mott insulator, Nature (London) 393, 550 (1998).

[7] S. Chakravarty, R. B. Laughlin, D. K. Morr, and C. Nayak, Hidden order in the cuprates, Phys. Rev. B 63, 094503 (2001).

[8] A. Kaminski, S. Rosenkranz, H. Fretwell, J. Campuzano, Z. Li, H. Raffy, W. Cullen, H. You, C. Olson, C. Varma et al., Spontaneous breaking of time-reversal symmetry in the pseudogap state of a high-tc superconductor, Nature (London) 416, 610 (2002)

[9] M. E. Simon and C. M. Varma, Detection and Implications of a Time-Reversal Breaking State in Underdoped Cuprates, Phys. Rev. Lett. 89, 247003 (2002).

[10] M. S. Scheurer, S. Chatterjee, W. Wu, M. Ferrero, A. Georges, and S. Sachdev, Topological order in the pseudogap metal, Proc. Natl. Acad. Sci. USA 115, E3665 (2018).

[11] M. S. Scheurer and S. Sachdev, Orbital currents in insulating and doped antiferromagnets, Phys. Rev. B 98, 235126 (2018).

[12] S. Sachdev, Topological order, emergent gauge fields, and fermi surface reconstruction, Rep. Prog. Phys. 82, 014001 (2019).

[13] Z. L. Weng, D. N. Sheng, and C. S. Ting, Mean-field description of the phase string effect in the t-j model, Phys. Rev. B 59, 8943 (1999).

[14] T. D. Stanescu and G. Kotliar, Fermi arcs and hidden zeros of the green function in the pseudogap state, Phys. Rev. B 74, 125110 (2006).
[15] P. Phillips, Colloquium: Identifying the propagating charge modes in doped mott insulators, Rev. Mod. Phys. 82, 1719 (2010).

[16] J. Zaanen and B. Overbosch, Mottness collapse and statistical quantum criticality, Philos. Trans. R. Soc. London, Ser. A 369, 1599 (2011).

[17] M. Imada, Y. Yamaji, S. Sakai, and Y. Motome, Theory of pseudogap and superconductivity in doped mott insulators, Ann. Phys. 523, 629 (2011).

[18] E. Dagotto, Correlated electrons in high-temperature superconductors, Rev. Mod. Phys. 66, 763 (1994).

[19] D. J. Scalapino, Numerical studies of the 2D Hubbard model, in Handbook of High-Temperature Superconductivity: Theory and Experiment, edited by J. R. Schrieffer and J. S. Brooks (Springer, New York, 2007), pp. 495-526.

[20] J. Jaklič and P. Prelovšek, Finite-temperature properties of doped antiferromagnets, Adv. Phys. 49, 1 (2000).

[21] K. Haule, A. Rosch, J. Kroha, and P. Wölfle, Pseudogaps in the $t-j$ model: An extended dynamical mean-field theory study, Phys. Rev. B 68, 155119 (2003).

[22] N. Bulut, D. J. Scalapino, and S. R. White, One-Electron Spectral Weight of the Doped Two-Dimensional Hubbard Model, Phys. Rev. Lett. 72, 705 (1994).

[23] R. Preuss, W. Hanke, C. Gröber, and H. G. Evertz, Pseudogaps and their Interplay with Magnetic Excitations in the Doped 2D Hubbard Model, Phys. Rev. Lett. 79, 1122 (1997).

[24] W. Wu, M. Ferrero, A. Georges, and E. Kozik, Controlling Feynman diagrammatic expansions: Physical nature of the pseudogap in the two-dimensional Hubbard model, Phys. Rev. B 96, 041105(R) (2017).

[25] M. Kohno, Mott Transition in the Two-Dimensional Hubbard Model, Phys. Rev. Lett. 108, 076401 (2012).

[26] A. Georges, G. Kotliar, W. Krauth, and M. J. Rozenberg, Dynamical mean-field theory of strongly correlated fermion systems and the limit of infinite dimensions, Rev. Mod. Phys. 68, 13 (1996).

[27] T. A. Maier, M. Jarrell, T. Prushke, and M. H. Hettler, Quantum cluster theories, Rev. Mod. Phys. 77, 1027 (2005).

[28] S. Sakai, Y. Motome, and M. Imada, Evolution of Electronic Structure of Doped Mott Insulators: Reconstruction of Poles and Zeros of Green's Function, Phys. Rev. Lett. 102, 056404 (2009). 
[29] E. Gull, O. Parcollet, and A. J. Millis, Superconductivity and the Pseudogap in the Two-Dimensional Hubbard Model, Phys. Rev. Lett. 110, 216405 (2013).

[30] O. Gunnarsson, T. Schäfer, J. P. F. LeBlanc, E. Gull, J. Merino, G. Sangiovanni, G. Rohringer, and A. Toschi, Fluctuation Diagnostics of the Electron Self-Energy: Origin of the Pseudogap Physics, Phys. Rev. Lett. 114, 236402 (2015).

[31] W. Wu, M. S. Scheurer, S. Chatterjee, S. Sachdev, A. Georges, and M. Ferrero, Pseudogap and Fermi-Surface Topology in the Two-Dimensional Hubbard Model, Phys. Rev. X 8, 021048 (2018).

[32] O. Cyr-Choinière, R. Daou, F. Laliberté, C. Collignon, S. Badoux, D. LeBoeuf, J. Chang, B. J. Ramshaw, D. A. Bonn, W. N. Hardy, R. Liang, J.-Q. Yan, J.-G. Cheng, J.-S. Zhou, J. B. Goodenough, S. Pyon, T. Takayama, H. Takagi, N. Doiron-Leyraud, and L. Taillefer, Pseudogap temperature $T^{*}$ of cuprate superconductors from the Nernst effect, Phys. Rev. B 97, 064502 (2018).

[33] A. Kampf and J. R. Schrieffer, Pseudogaps and the spin-bag approach to high- $T_{c}$ superconductivity, Phys. Rev. B 41, 6399 (1990).

[34] A. P. Kampf and J. R. Schrieffer, Spectral function and photoemission spectra in antiferromagnetically correlated metals, Phys. Rev. B 42, 7967 (1990).

[35] Y. M. Vilk and A. -M. S. Tremblay, Non-perturbative manybody approach to the Hubbard model and single-particle pseudogap, J. Phys. I 7, 1309 (1997).

[36] B. Kyung, V. Hankevych, A.-M. Daré, and A.-M. S. Tremblay, Pseudogap and Spin Fluctuations in the Normal State of the Electron-Doped Cuprates, Phys. Rev. Lett. 93, 147004 (2004).

[37] Y. F. Wang, C. D. Gong, and S. Y. Zhu, Field-induced gap, pseudogap and new van hove singularity in the triangular lattice, Europhys. Lett. 69, 404 (2005).

[38] D. Sen and R. Chitra, Large-U limit of a Hubbard model in a magnetic field: Chiral spin interactions and paramagnetism, Phys. Rev. B 51, 1922 (1995).

[39] Y.-H. Zhang and T. Senthil, Bridging Hubbard model physics and quantum Hall physics in trilayer
graphene/h-BN moiré superlattice, Phys. Rev. B 99, 205150 (2019).

[40] C. Schrade and L. Fu, Spin-valley density wave in moiré materials, Phys. Rev. B 100, 035413 (2019).

[41] H. T. Dang, X. Y. Xu, K.-S. Chen, Z. Y. Meng, and S. Wessel, Mott transition in the triangular lattice Hubbard model: A dynamical cluster approximation study, Phys. Rev. B 91, 155101 (2015).

[42] T. Shirakawa, T. Tohyama, J. Kokalj, S. Sota, and S. Yunoki, Ground-state phase diagram of the triangular lattice Hubbard model by the density-matrix renormalization group method, Phys. Rev. B 96, 205130 (2017).

[43] A. Szasz, J. Motruk, M. P. Zaletel, and J. E. Moore, Observation of a Chiral Spin Liquid Phase of the Hubbard Model on the Triangular Lattice: A Density Matrix Renormalization Group Study, Phys. Rev. X 10, 021042 (2020).

[44] D. Galanakis, T. D. Stanescu, and P. Phillips, Mott transition on a triangular lattice, Phys. Rev. B 79, 115116 (2009).

[45] B. Kyung, Electronic properties of the Hubbard model on a frustrated triangular lattice, Phys. Rev. B 75, 033102 (2007).

[46] M. Civelli, M. Capone, S. S. Kancharla, O. Parcollet, and G. Kotliar, Dynamical Breakup of the Fermi Surface in a Doped Mott Insulator, Phys. Rev. Lett. 95, 106402 (2005).

[47] D. Sénéchal and A.-M. S. Tremblay, Hot Spots and Pseudogaps for Hole- and Electron-Doped High-Temperature Superconductors, Phys. Rev. Lett. 92, 126401 (2004).

[48] T. A. Maier, T. Pruschke, and M. Jarrell, Angle-resolved photoemission spectra of the Hubbard model, Phys. Rev. B 66, 075102 (2002).

[49] K. Yoshitaka, S. Kazuhiro, E. Yusuke, S. Yoshiaki, P. Jiang, T. Taishi, Y. Seiji, M. Y. Hiroshi, and K. Reizo, Electron-hole doping asymmetry of fermi surface reconstructed in a simple mott insulator, Nat. Commun. 7, 12356 (2016).

[50] Y. Yu, L. Ma, P. Cai, R. Zhong, C. Ye, J. Shen, G. D. Gu, X. H. Chen, and Y. Zhang, High-temperature superconductivity in monolayer $\mathrm{Bi}_{2} \mathrm{Sr}_{2} \mathrm{CaCu}_{2} \mathrm{O}_{8+\delta}$, Nature (London) 575, 156 (2019). 